\title{
THE GEOMETRY UNDERLYING MIRROR SYMMETRY
}

\author{
DAVID R. MORRISON
}

\begin{abstract}
The recent result of Strominger, Yau and Zaslow relating mirror symmetry to the quantum field theory notion of T-duality is reinterpreted as providing a way of geometrically characterizing which Calabi-Yau manifolds have mirror partners. The geometric description - that one Calabi-Yau manifold should serve as a compactified, complexified moduli space for special Lagrangian tori on the other Calabi-Yau manifold - is rather surprising. We formulate some precise mathematical conjectures concerning how these moduli spaces are to be compactified and complexified, as well as a definition of geometric mirror pairs (in arbitrary dimension) which is independent of those conjectures. We investigate how this new geometric description ought to be related to the mathematical statements which have previously been extracted from mirror symmetry. In particular, we discuss how the moduli spaces of the 'mirror' Calabi-Yau manifolds should be related to one another, and how appropriate subspaces of the homology groups of those manifolds could be related. We treat the case of K3 surfaces in some detail.
\end{abstract}

Precise mathematical formulations of the string theory phenomenon known as "mirror symmetry" [21, 33, 19, 27] have proved elusive up until now, largely due to one of the more mysterious aspects of that symmetry: as traditionally formulated, mirror symmetry predicts an equivalence between physical theories associated to certain pairs of Calabi-Yau manifolds, but does not specify any geometric relationship between those manifolds. However, such a geometric relationship has recently been discovered in a beautiful paper of Strominger, Yau and Zaslow 53. Briefly put, these authors find that the mirror partner $X$ of a given Calabi-Yau threefold $Y$ should be realized as the (compactified and complexified) moduli space for special Lagrangian tori on $Y$.

This relationship was derived in [53] from the assumption that the physical theories associated to the pair of Calabi-Yau threefolds satisfy a strong property called "quantum mirror symmetry" [52, 6, 12, 42, In the present paper, we will invert the logic, and use this geometric relationship as a characterization of mirror pairs, which we formulate in arbitrary dimension. On the one hand, this characterization can be stated in purely

\footnotetext{
${ }^{1}$ Our definition appears to produce valid mirror pairs of conformal field theories in any dimension, even though the string-theoretic arguments of [53] cannot be directly
} 
mathematical terms, providing a criterion by which mathematicians can recognize mirror pairs. On the other hand, the characterization contains the essential ingredients needed to apply the quantum field theory argument known as "T-duality" which could in principle establish the equivalence of the associated string theories at the level of physical rigor (cf. [45, 53]). If This geometric characterization thus appears to capture the essence of mirror symmetry in mathematical terms.

This paper is organized as follows. In section 1, we give a brief summary of quantum mirror symmetry and review the derivation of the geometric relationship given in [53]. In section 2, we discuss the theory of special Lagrangian submanifolds [29] and their moduli spaces [37], and explain how these moduli spaces should be compactified and complexified (following [53]). In section 3, we review in detail the topological and Hodge-theoretic properties which have formed the basis for previous mathematical discussions of mirror symmetry. We then formulate in section 4 our characterization of geometric mirror pairs, which we (conjecturally) relate to those topological and Hodge-theoretic properties. In section 5 we present some new results concerning the geometric mirror relationship, including a discussion of how it leads to a connection between certain subspaces of $H_{n}(Y)$ and $H_{\text {even }}(X)$, and in section 6 we discuss geometric mirror symmetry for K3 surfaces.

\section{QUANTUM MIRROR SYMMETRY}

Moduli spaces which occur in physics often differ somewhat between the classical and quantum versions of the same theory. For example, the essential mathematical data needed to specify the two-dimensional conformal field theory associated to a Calabi-Yau manifold $X$ consists of a Ricci-flat metric $g_{i j}$ on $X$ and an $\mathbb{R} / \mathbb{Z}$-valued harmonic 2-form $B \in \mathcal{H}^{2}(X, \mathbb{R} / \mathbb{Z})$. The classical version of this theory is independent of $B$ and invariant under rescaling the metric; one might thus call the set of all diffeomorphism classes of Ricci-flat metrics of fixed volume on $X$ the "classical moduli space" of the theory. The volume of the metric and the 2 -form $B$ must be included in the moduli space once quantum effects are taken into account; in a "semiclassical approximation" to the quantum moduli space, one treats the data $\left(g_{i j}, B\right)$ (modulo diffeomorphism) as providing a complete description of that space. However, a closer analysis of the physical theory reveals that this is indeed only an

extended to arbitrary dimension to conclude that all mirror pairs ought to arise in this fashion.

${ }^{2}$ There are some additional details which need to be understood before this can be regarded as fully established in physics. 
approximation to the quantum moduli space, with the necessary modifications becoming more and more significant as the volume is decreased. The ultimate source of these modifications - which are of a type referred to as "nonperturbative" in physics - is the set of holomorphic curves on $X$ and their moduli spaces. A convenient mathematical way of describing how these modifications work is this: there are certain "correlation functions" of the physical theory, which are described near the large volume limit as power series whose coefficients are determined by the numbers of holomorphic 2-spheres on $X$. The quantum moduli space should then be identified as the natural domain of definition for these correlation functions. To construct it starting from the semiclassical approximation, one first restricts to the open set in which the power series converge, and then extends by analytic continuation to find the complete moduli space. The refer to this space as the quantum conformal field theory moduli space $\mathcal{M}_{C F T}(X)$. (When necessary, we use the notation $\mathcal{M}_{C F T}^{s c}(X)$ to refer to the semiclassical approximation to this space.)

A similar story has emerged within the last year concerning the moduli spaces for type IIA and IIB string theories compactified on a Calabi-Yau threefold $X$. The classical low-energy physics derived from these string theories is determined by a quantum conformal field theory, so one might think of the quantum conformal field theory moduli space described above as being a "classical moduli space" for these theories.

In the semiclassical approximation to the quantum moduli spaces of these string theories, we encounter additional mathematical data which must be specified. In the case of the IIA theory, the new data consist of a choice of a nonzero complex number (called the "axion/dilaton expectation value"), together with an $\mathbb{R} / \mathbb{Z}$-valued harmonic 3 -form $C \in$ $\mathcal{H}^{3}(X, \mathbb{R} / \mathbb{Z})$. This last object has a familiar mathematical interpretation as a point in the intermediate Jacobian of $X$ (taking a complex structure on $X$ for which the metric is Kähler). In the case of the IIB theory on a Calabi-Yau threefold $Y$, the corresponding new data are a choice of nonzero "axion-dilaton expectation value" as before, together with what we might call a quantum $\mathbb{R} / \mathbb{Z}$-valued harmonic even class $C \in \mathcal{H}_{Q}^{\text {even }}(Y, \mathbb{R} / \mathbb{Z})$. The word "quantum" and the subscript " $Q$ " here refer to the fact that we must use the quantum cohomology lattice rather

\footnotetext{
${ }^{3}$ There are several possible (equivalent) mathematical interpretations which can be given to these correlation functions: they can be interpreted as defining a new ring structure on the cohomology (defining the so-called quantum cohomology ring) or they can be regarded as defining a variation of Hodge structure over the moduli space. We will review this in more detail in section 3 below.

${ }^{4}$ There can also be modifications caused by higher genus curves [5], but these are less drastic and are not important for our purposes here.
} 
than the ordinary cohomology lattice in determining when two harmonic $C$ 's are equivalent. (The details of this difference are not important here; we refer the interested reader to [6, 42].) For both the IIA and IIB theories, a choice of such "data" as above can be used to describe a low-energy supergravity theory in four dimensions.

Just as in the earlier example, there are additional corrections to the semiclassical description of the moduli space coming from "nonperturbative effects" [52, 26, 12], among which are some which go by the name of "Dirichlet-branes," or "D-branes" for short. The source of these Dbrane corrections differs for the two string theories we are considering: in the type IIA theory, they come from moduli spaces of algebraic cycles on $X$ equipped with flat $U(1)$-bundles, or more generally, from moduli spaces of coherent sheaves on $X$. In the type IIB theory, the D-brane corrections come from (complexified) moduli spaces of so-called supersymmetric 3-cycles on $Y$, the mathematics of which will be described in the next section. Just as the correlation functions which we could use to determine the structure of the quantum conformal field theory moduli space involved a series expansion with contributions from the holomorphic spheres, the correlation functions in this theory will receive contributions from the coherent sheaves or supersymmetric 3-cycles, with the precise nature of the contribution arising from an integral over the corresponding moduli space.

Quantum mirror symmetry is the assertion that there should exist pairs of Calabi-Yau threefolds $(X, Y)$ such that the type IIA string theory compactified on $X$ is isomorphic to the type IIB string theory compactified on $Y$; there should be compatible isomorphisms of both the classical and quantum theories. The isomorphism of the classical theories is the statement that the corresponding (quantum-corrected) conformal field theories should be isomorphic. This is the version of mirror symmetry which was translated into mathematical terms some time ago, and leads to the surprising statements relating the quantum cohomology on $X$ to the geometric variation of Hodge structure on $Y$ (and vice versa).

\footnotetext{
${ }^{5} \mathrm{~A}$ D-brane in type IIA theory is ordinarily described as a complex submanifold $Z$ together with a flat $U(1)$-bundle on that submanifold; the associated holomorphic line bundle on $Z$ can be extended by zero to give a coherent sheaf on $X$. The arbitrary coherent sheaves which we consider here correspond to what are called "bound states of D-branes" in physics. (This same observation has been independently made by Maxim Kontsevich, and by Jeff Harvey and Greg Moore.)

${ }^{6}$ There are versions of quantum mirror symmetry which can be formulated in other (low) dimensions, but since these are statements about compactifying ten-dimensional string theories, they cannot be extended to arbitrarily high dimension.
} 
On the other hand, the isomorphism of the quantum theories has only recently been explored.] At the semiclassical level, one infers isomorphisms between the intermediate Jacobian of $X$ (the 3 -form discussed above), and an analogue of that intermediate Jacobian in quantum cohomology of $Y$. The full quantum isomorphism would involve properties of the coherent sheaves on $X$, as related to the supersymmetric 3 -cycles on $Y$. In fact, there should be enough correlation functions in the quantum theory to fully measure the structure of the individual moduli spaces of these sheaves and cycles, so we should anticipate that the moduli spaces themselves are isomorphic. If It is this observation which was the key to the Strominger-Yau-Zaslow argument.

Strominger, Yau and Zaslow observe that the algebraic 0-cycles of length one on $X$ (which can be thought of as torsion sheaves supported at a single point) have as their moduli space $X$ itself. According to quantum mirror symmetry, then, there should be a supersymmetric 3-cycle $M$ on $Y$ with precisely the same moduli space, that is, the moduli space of $M$ should be $X$. Since the complex dimension of the moduli space is three, it follows from a result of McLean [37] (see the next section) that $b_{1}(M)=3$. Now as we will explain in the next section, the complexified moduli space $\widehat{X}$ for the supersymmetric 3 -cycles parameterizes both the choice of 3 -cycle $M$ and the choice of a flat $\mathrm{U}(1)$-bundle on $M$. Fixing the cycle but varying the bundle gives a real 3 -torus on $\widehat{X}$ (since $b_{1}(M)=3$ ), which turns out to be a supersymmetric cycle on that space. This is the "inverse" mirror transform, based on a cycle $\widetilde{M}$ which is in fact a 3-torus. Thus, by applying mirror symmetry twice if necessary, we see that we can - without loss of generality - take the original supersymmetric 3 -cycle $M$ to be a 3 -torus. In this case, we say that $Y$ has a supersymmetric $T^{3}$-fibration; note that singular fibers must in general be allowed in such fibrations.

We have thus obtained the rough geometric characterization of the pair $(X, Y)$ which was stated in the introduction: $X$ should be the moduli space for supersymmetric 3-tori on $Y$. This characterization is "rough"

\footnotetext{
${ }^{7}$ The speculation some time ago by Donagi and Markman 22 that some sort of Fourier transform should relate the continuous data provided by the intermediate Jacobian to the discrete data provided by the holomorphic curves is closely related to these isomorphisms of quantum theories.

${ }^{8}$ In the case of coherent sheaves, one should not use the usual moduli spaces from algebraic geometry, but rather some sort of "virtual fundamental cycle" on the algebrogeometric moduli space, whose dimension coincides with the "expected dimension" of the algebro-geometric moduli space as computed from the Riemann-Roch theorem. When the moduli problem is unobstructed, this virtual fundamental cycle should coincide with the usual fundamental cycle on the algebro-geometric moduli space.
} 
due to technical difficulties involving both the compactifications of these moduli spaces, and the complex structures on them. We will take a different path in section 4 below, and give a precise geometric characterization which sidesteps these issues.

This line of argument can be pushed a bit farther, by considering the algebraic 3-cycle on $X$ in the fundamental class equipped with a flat $U(1)$ bundle (which must be trivial, and corresponds to the coherent sheaf $\mathcal{O}_{X}$ ). There is precisely one of these, so we find a moduli space consisting of a single point. Its mirror should then be a supersymmetric 3-cycle $M^{\prime}$ with $b_{1}\left(M^{\prime}\right)=0$. Moreover, we should expect quantum mirror symmetry to preserve the intersection theory of the cycles represented by D-branes (up to sign), so since the 0 -cycle and 6-cycle on $X$ have intersection number one, we should expect $M$ and $M^{\prime}$ to have intersection number one if $M^{\prime}$ is oriented properly. In other words, the supersymmetric $T^{3}$-fibration on $Y$ should have a section, f and the base of the fibration should satisfy $b_{1}=0$.

The final step in the physics discussion given in [53] is to observe that given a Calabi-Yau threefold with a supersymmetric $T^{3}$-fibration and a mirror partner, the mirror partner can be recovered by dualizing the tori in the fibration, at least generically. This suggests that by applying an appropriate duality transformation to the path integral - this is known as the "T-duality argument" in quantum field theory - one should be able to conclude that mirror symmetry does indeed hold for the corresponding physical theories. Strominger, Yau and Zaslow take the first steps towards constructing such an argument, at appropriate limit points of the moduli space. To complete the argument and extend it to general points in the moduli space, one would need to understand the behavior of the T-duality transformations near the singular fibers; to this end, a detailed mathematical study of the possible singular fibers is needed. Some preliminary information about these singularities can be found in [29, 17] (see also [28]).

\section{Moduli of SPECIAL LAGRANGIAN SUBMANIFOLDS}

The structure of the supersymmetric 3-cycles which played a rôle in the previous section was determined in [12], where it was found that they are familiar mathematical objects known as special Lagrangian submanifolds. These are a particular class of submanifolds of Calabi-Yau manifolds first studied by Harvey and Lawson [29]. We proceed to the definitions.

\footnotetext{
${ }^{9}$ The existence of a section is also expected on other grounds: the set of flat $U(1)$ bundles on $M$ has a distinguished element - the trivial bundle. This provides a section for the "dual" fibration, and suggests (by a double application of quantum mirror symmetry as above) that the original fibration could have been chosen to have a section, without loss of generality.
} 
A Calabi-Yau manifold is a compact connected orientable manifold $Y$ of dimension $2 n$ which admits Riemannian metrics whose (global) holonomy is contained in $\mathrm{SU}(n)$. For any such metric, there is a complex structure on the manifold with respect to which the metric is Kähler, and a nowhere-vanishing holomorphic $n$-form $\Omega$ (unique up to constant multiple). The complex structure, the $n$-form $\Omega$ and the Kähler form $\omega$ are all covariant constant with respect to the Levi-Civita connection of the Riemannian metric. This implies that the metric is Ricci-flat, and that $\Omega \wedge \bar{\Omega}$ is a constant multiple of $\omega^{n}$.

A special Lagrangian submanifold of $Y$ is a compact real $n$-manifold $M$ together with an immersion $f: M \rightarrow Y$ such that $f^{*}\left(\Omega_{0}\right)$ coincides with the induced volume form $d \mathrm{vol}_{M}$ for an appropriate choice of holomorphic $n$-form $\Omega_{0}$. Equivalently [29], one can require that (1) $M$ is a Lagrangian submanifold with respect to the symplectic structure defined by $\omega$, i.e., $f^{*}(\omega)=0$, and $(2) f^{*}\left(\operatorname{Im} \Omega_{0}\right)=0$ for an appropriate $\Omega_{0}$. To state this second condition in a way which does not require that $\Omega_{0}$ be specified, write an arbitrary holomorphic $n$-form $\Omega$ in the form $\Omega=c \Omega_{0}$, and note that

$$
\int_{M} f^{*}(\Omega)=c \int_{M} f^{*}\left(\Omega_{0}\right)=c(\operatorname{vol} M)
$$

Thus, the "appropriate" $n$-form is given by

$$
\Omega_{0}=\frac{(\operatorname{vol} M) \Omega}{\int_{M} f^{*}(\Omega)}
$$

and we can replace condition (2) by

$$
f^{*}\left(\operatorname{Im}\left(\frac{\Omega}{\int_{M} f^{*}(\Omega)}\right)\right)=0 .
$$

(The factor of vol $M$ is a real constant which can be omitted from this last condition.)

Very few explicit examples of special Lagrangian submanifolds are known. (This is largely due to our lack of detailed understanding of the Calabi-Yau metrics themselves.) One interesting class of examples due to Bryant 18 comes from Calabi-Yau manifolds which are complex algebraic varieties defined over the real numbers: the set of real points on the Calabi-Yau manifold is a special Lagrangian submanifold. Another interesting class of examples is the special Lagrangian submanifolds of a K3 surface, which we will discuss in section 6 .

In general, special Lagrangian submanifolds can be deformed, and there will be a moduli space which describes the set of all special Lagrangian submanifolds in a given homology class. Given a special Lagrangian $f$ : 
$M \rightarrow Y$ and a deformation of the map $f$, since $f^{*}(\omega)=0$, the almostcomplex structure on $Y$ induces a canonical identification between the normal bundle of $M$ in $Y$ and the tangent bundle of $M$. Thus, the normal vector field defined by the deformation can be identified with a 1-form on $Y$.

The key result concerning the moduli space is due to McLean.

Theorem (McLean [37]).

1. First-order deformations of $f$ are canonically identified with the space of harmonic 1-forms on $Y$.

2. All first-order deformations of $f: M \rightarrow Y$ can be extended to actual deformations. In particular, the moduli space $\mathcal{M}_{s L}(M, Y)$ of special Lagrangian maps from $M$ to $Y$ is a smooth manifold of dimension $b_{1}(M)$.

(We have in mind a global structure on $\mathcal{M}_{s L}(M, Y)$ in which two maps will determine the same point in the moduli space if they differ by a diffeomorphism of $Y$.) McLean also observes that $\mathcal{M}=\mathcal{M}_{s L}(M, Y)$ admits a natural $n$-form $\Theta$ defined by

$$
\Theta\left(v_{1}, \ldots, v_{n}\right)=\int_{M} \theta_{1} \wedge \cdots \wedge \theta_{n}
$$

where $\theta_{j}$ is the harmonic 1 -form associated to $v_{j} \in T_{\mathcal{M}, f}$.

As was implicitly discussed in the last section, the moduli spaces of interest in string theory contain additional pieces of data. To fully account for the "nonperturbative D-brane effects" in the physical theory (when $n=3$ ), the moduli space which we integrate over must include not only the choice of special Lagrangian submanifold, but also a choice of flat $\mathrm{U}(1)$-bundle on that manifold. If we pick a point $b$ on a manifold $M$, then the space of flat $\mathrm{U}(1)$-bundles on $M$ is given by

$$
\operatorname{Hom}\left(\pi_{1}(M, b), \mathrm{U}(1)\right) \cong H^{1}(M, \mathbb{R}) / H^{1}(M, \mathbb{Z})
$$

Thus, if we construct a universal family for our special Lagrangian submanifold problem, i.e., a diagram

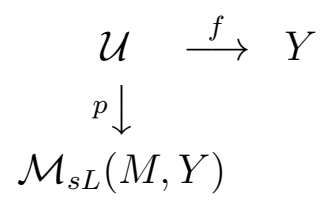

with the property that the fibers of $p$ are diffeomorphic to $M$ and $\left.f\right|_{p^{-1}(m)}$ is the map labeled by $m$, and if $p$ has a section $s: \mathcal{M}_{s L}(M, Y) \rightarrow \mathcal{U}$, then we can define a moduli space which includes the data of a flat $\mathrm{U}(1)$-bundle by

$$
\mathcal{M}_{D}(M, Y):=R^{1} p_{*} \mathbb{R}_{\mathcal{U}} / R^{1} p_{*} \mathbb{Z}_{\mathcal{U}}
$$


which at each point $m \in \mathcal{M}_{s L}(M, Y)$ specializes to

$$
H^{1}\left(p^{-1}(m), \mathbb{R}\right) / H^{1}\left(p^{-1}(m), \mathbb{Z}\right) \cong \operatorname{Hom}\left(\pi_{1}\left(p^{-1}(m), s(m)\right), \mathrm{U}(1)\right) .
$$

(In the case $n=3$, this is the "D-brane" moduli space, which motivates our notation.) Note that this space fibers naturally over $\mathcal{M}_{s L}(M, Y)$, and that there is a section of the fibration, given by the trivial U(1)-bundles.

Both the base and the fiber of the fibration $\mathcal{M}_{D}(M, Y) \rightarrow \mathcal{M}_{s L}(M, Y)$ have dimension $b_{1}(M)$, and the fibers are real tori. In fact, we expect from the physics that there will be a family of complex structures on $\mathcal{M}_{D}(M, Y)$ making it into a complex manifold of complex dimension $b_{1}(M)$. The real tori should roughly correspond to subspaces obtained by varying the arguments of the complex variables while keeping their norms fixed. It is expected from the physics that the complex structure should depend on the choice of both a Ricci-flat metric on $Y$ and also on an auxiliary harmonic 2-form $B$. (This would make $\mathcal{M}_{D}(M, Y)$ into a "complexification" of the moduli space $\mathcal{M}_{s L}(M, Y)$ as mentioned in the introduction.) It it not clear at present precisely how those complex structures are to be constructed, although in the case $b_{1}(M)=n$, a method is sketched in [53] for producing an asymptotic formula for the Ricci-flat metric which would exhibit the desired dependence on $g_{i j}$ and $B$, and the first term in that formula is calculated. The complex structure could in principle be inferred from the metric if it were known.

Motivated by the Strominger-Yau-Zaslow analysis, we now turn our attention to the case in which $M$ is an $n$-torus. The earliest speculations that the special Lagrangian $n$-tori might play a distinguished rôle in studying Calabi-Yau manifolds were made by McLean [37, who pointed out that if $M=T^{n}$ then the deformations of $M$ should locally foliate $Y$. (There should be no self-intersections nearby since the harmonic 1forms corresponding to the first-order deformations are expected to have no zeros if the metric on the torus is close to being flat.) McLean speculated that - by analogy with the K3 case where such elliptic fibrations are well-understood - if certain degenerations were allowed, the deformations of $M$ might fill out the whole of $Y$. We formulate this as a conjecture (essentially due to McLean).

Conjecture 1. Suppose that $f: T^{n} \rightarrow Y$ is a special Lagrangian n-torus. Then there is a natural compactification $\overline{\mathcal{M}}_{s L}\left(T^{n}, Y\right)$ of the moduli space

\footnotetext{
${ }^{10}$ In the language of [53], the "tree-level" metric on the moduli space is computed, but the instanton corrections to that tree-level metric are left unspecified.
} 


$$
\begin{gathered}
\mathcal{M}_{s L}\left(T^{n}, Y\right) \text { and a proper map } g: Y \rightarrow \overline{\mathcal{M}}_{s L}\left(T^{n}, Y\right) \text { such that } \\
\qquad g^{-1}\left(\mathcal{M}_{s L}(M, Y)\right) \hookrightarrow Y \\
g \downarrow \\
\mathcal{M}_{s L}(M, Y)
\end{gathered}
$$

is a universal family of Lagrangian $n$-tori in the same homology class as $f$.

Definition 1. When the properties in conjecture 1 hold, we say that $Y$ has a special Lagrangian $T^{n}$-fibration.

It is not clear at present what sort of structure should be required of $\overline{\mathcal{M}}_{s L}\left(T^{n}, Y\right)$ : perhaps it should be a manifold with corners, $\square$ or perhaps some more exotic singularities should be allowed in the compactification. We will certainly want to require that the complex structures extend to the compactification, and that the section of the fibration extend to a $\operatorname{map} \overline{\mathcal{M}}_{s L}\left(T^{n}, Y\right) \rightarrow Y$.

The mirror symmetry analysis of [53] as reviewed in the previous section suggests that the family of dual tori $\mathcal{M}_{D}\left(T^{n}, Y\right)$ can also be compactified, resulting in a space which is itself a Calabi-Yau manifold. We formalize this as a conjecture as well.

Conjecture 2. The family $\mathcal{M}_{D}\left(T^{n}, Y\right)$ of dual tori over $\mathcal{M}_{s L}\left(T^{n}, Y\right)$ can be compactified to a manifold $X$ with a proper map $\gamma: X \rightarrow \overline{\mathcal{M}}_{s L}\left(T^{n}, Y\right)$, such that $X$ admits metrics with $\mathrm{SU}(n)$ holonomy for which the fibers of $\left.\gamma\right|_{\gamma^{-1}\left(\mathcal{M}_{s L}\left(T^{n}, Y\right)\right)}$ are special Lagrangian $n$-tori. Moreover, the fibration $\gamma$ admits a section $\tau: \overline{\mathcal{M}}_{s L}\left(T^{n}, Y\right) \rightarrow X$ such that $\tau\left(\mathcal{M}_{s L}\left(T^{n}, Y\right)\right) \subset$ $\mathcal{M}_{D}\left(T^{n}, Y\right) \subset X$ is the zero-section.

It seems likely that for an appropriate holomorphic $n$-form $\Omega_{0}$ on $X$, the pullback $\tau^{*}\left(\Omega_{0}\right)$ will coincide with McLean's $n$-form $\Theta$ when restricted to $\mathcal{M}_{s L}\left(T^{n}, Y\right)$.

The most accessible portion of these conjectures would be the following:

Sub-Conjecture . The family $\mathcal{M}_{D}\left(T^{n}, Y\right)$ of dual tori over $\mathcal{M}_{s L}\left(T^{n}, Y\right)$ admits complex structures and Ricci-flat Kähler metrics. In particular, it has a nowhere vanishing holomorphic n-form.

Strominger, Yau and Zaslow have obtained some partial results concerning this subconjecture, for which we refer the reader to [53]. It appears, for example, that the construction of the complex structure on

\footnotetext{
${ }^{11}$ This possibility is suggested by the structure of toric varieties, the moment maps for which express certain complex manifolds as $T^{n}$-fibrations over manifolds with corners (compact convex polyhedra).
} 
the D-brane moduli space should be local around each torus in the torus fibration.

\section{MATHEMATICAL CONSEQUENCES OF MIRROR SYMMETRY}

There is by now quite a long history of extracting mathematical statements from the physical notion of mirror symmetry. Many of these work in arbitrary dimension, where there is evidence in physics for mirror symmetry among conformal field theories [27, 25]. एా In this section, we review two of those mathematical statements, presented here as definitions. As the discussion is a bit technical, some readers may prefer to skip to the next section, where we formulate our new definition of geometric mirror pairs inspired by the Strominger-Yau-Zaslow analysis. Throughout this section, we let $X$ and $Y$ be Calabi-Yau manifolds of dimension $n$.

The first prediction one extracts from physics about a mirror pair is a simple equality of Hodge numbers.

Definition 2. We say that the pair $(X, Y)$ passes the topological mirror test if $h^{n-1,1}(X)=h^{1,1}(Y)$ and $h^{1,1}(X)=h^{n-1,1}(Y)$.

Many examples of pairs passing this test are known; indeed, the observation of this "topological pairing" in a class of examples was one of the initial pieces of evidence in favor of mirror symmetry [19]. Subsequent constructions of Batyrev and Borisov [8, 16, 9] show that all Calabi-Yau complete intersections in toric varieties belong to pairs which pass this topological mirror test.

For simply-connected Calabi-Yau threefolds, the Hodge numbers $h^{1,1}$ and $h^{n-1,1}$ determine all of the others, but in higher dimension there are more. Naïvely one expects to find that $h^{p, q}(X)=h^{n-p, q}(Y)$. However, as was discovered by Batyrev and collaborators [11, 10], the proper interpretation of the numerical invariants of the physical theories requires a modified notion of "string-theoretic Hodge numbers" $h_{s t}^{p, q}$; once this modification has been made, these authors show that $h_{s t}^{p, q}(X)=h_{s t}^{n-p, q}(Y)$ for the Batyrev-Borisov pairs $(X, Y)$ of complete intersections in toric varieties. The class of pairs for which this modification is needed includes some of those given by the Greene-Plesser construction [27] for which mirror symmetry of the conformal field theories has been firmly established in physics, so it would appear that this modification is truly necessary for a mathematical interpretation of mirror symmetry. Hopefully, it too will follow from the geometric characterization being formulated in this paper.

\footnotetext{
${ }^{12}$ In low dimension where a string-theory interpretation is possible, this would become the "classical" mirror symmetry which one would also want to extend to a "quantum" mirror symmetry if possible.
} 
Going beyond the simple topological properties, a more precise and detailed prediction arises from identifying the quantum cohomology of one Calabi-Yau manifold with the geometric variation of Hodge structure of the mirror partner (in the case that the Calabi-Yau manifolds have no holomorphic 2-forms). We will discuss this prediction in considerable detail, in order to ensure that this paper has self-contained statements of the conjectures being proposed within it (particularly the ones in sections 4 and 5 below relating the "old" and "new" mathematical versions of mirror symmetry).

To formulate this precise prediction, let $X$ be a Calabi-Yau manifold with $h^{2,0}(X)=0$, and let $\widetilde{\mathcal{M}}_{C F T}^{s c}(X)$ be the moduli space of triples $\left(g_{i j}, B, \mathcal{J}\right)$ modulo diffeomorphism, where $\mathcal{J}$ is a complex structure for which the metric $g_{i j}$ is Kähler. The map $\widetilde{\mathcal{M}}_{C F T}^{s c}(X) \rightarrow \mathcal{M}_{C F T}^{s c}(X)$ is finite-to-one, so this is another good approximation to the conformal field theory moduli space. Moreover, there is a natural map $\widetilde{\mathcal{M}}_{C F T}^{s c}(X) \rightarrow$ $\mathcal{M}_{c x}(X)$ to the moduli space of complex structures on $X$, whose fiber over $\mathcal{J}$ is $\mathcal{K}_{\mathbb{C}}\left(X_{\mathcal{J}}\right) / \operatorname{Aut}\left(X_{\mathcal{J}}\right)$, where

$$
\mathcal{K}_{\mathbb{C}}\left(X_{\mathcal{J}}\right)=\left\{B+i \omega \in \mathcal{H}^{2}(X, \mathbb{C} / \mathbb{Z}) \mid \omega \in \mathcal{K}_{\mathcal{J}}\right\}
$$

is the complexified Kähler con $\mathrm{E}^{\Pi 3}$ of $X_{\mathcal{J}}\left(\mathcal{K}_{\mathcal{J}}\right.$ being its usual Kähler cone), and $\operatorname{Aut}\left(X_{\mathcal{J}}\right)$ is the group of holomorphic automorphisms of $X_{\mathcal{J}}$.

The moduli space of complex structures $\mathcal{M}_{c x}(X)$ has a variation of Hodge structure defined on it which is of geometric origin: roughly speaking, one takes a universal family $\pi: \mathcal{X} \rightarrow \mathcal{M}_{c x}(X)$ over the moduli space and constructs a variation of Hodge structure on the local system $R^{n} \pi_{*} \mathbb{Z}_{\mathcal{X}}$ by considering the varying Hodge decomposition of $H^{n}\left(X_{\mathcal{J}}, \mathbb{C}\right)$. The local system gives rise to a holomorphic vector bundle $\mathcal{F}:=\left(R^{n} \pi_{*} \mathbb{Z}_{\mathcal{X}}\right) \otimes$ $\mathcal{O}_{\mathcal{M}_{c x}(X)}$ with a flat connection $\nabla: \mathcal{F} \rightarrow \Omega_{\mathcal{M}_{c x}(X)}^{1} \otimes \mathcal{F}$ (whose flat sections are the sections of the local system), and the varying Hodge decompositions determine the Hodge filtration

$$
\mathcal{F}=\mathcal{F}^{0} \supset \mathcal{F}^{1} \supset \cdots \supset \mathcal{F}^{n} \supset\{0\}
$$

a filtration by holomorphic subbundles defined by

$$
\left.\mathcal{F}^{p}\right|_{\mathcal{J}}=H^{n, 0}\left(X_{\mathcal{J}}\right) \oplus \cdots \oplus H^{p, n-p}\left(X_{\mathcal{J}}\right),
$$

which is known to satisfy the Griffiths transversality property

$$
\nabla\left(\mathcal{F}^{p}\right) \subset \Omega_{\mathcal{M}_{c x}(X)}^{1} \otimes \mathcal{F}^{p-1} .
$$

Conversely, given the bundle with flat connection and filtration, the complexified local system $R^{n} \pi_{*} \mathbb{C}_{\mathcal{X}}$ can be recovered by taking (local) flat

\footnotetext{
${ }^{13}$ We are following the conventions of 40 rather than those of [38, 39.
} 
sections, and the Hodge structures can be reconstructed from the filtration. The original local system of $\mathbb{Z}$-modules is however additional data, and cannot be recovered from the bundle, connection and filtration alone.

The moduli space of complex structures $\mathcal{M}_{c x}(X)$ can be compactified to a complex space $\overline{\mathcal{M}}$, to which the bundles $\mathcal{F}^{p}$ and the connection $\nabla$ extend; however, the extended connection $\nabla$ acquires regular singular points along the boundary $\mathcal{B}$, which means that it is a map

$$
\nabla: \mathcal{F} \rightarrow \Omega^{1}(\log \mathcal{B}) \otimes \mathcal{F}
$$

The residues of $\nabla$ along boundary components describe the monodromy transformations about those components, the same monodromy which defines the local system. At normal crossings boundary points there is always an associated monodromy weight filtration, which we take to be a filtration on the homology group $H_{n}(X)$.

The data of the flat connection and the Hodge filtration are encoded in the conformal field theory on $X$ (at least for a sub-Hodge structure containing $\mathcal{F}^{n-1}$ ). 四 Since mirror symmetry reverses the rôles of base and fiber in the map

$$
\widetilde{\mathcal{M}}_{C F T}^{s c}(X) \rightarrow \mathcal{M}_{c x}(X)
$$

one of the predictions of mirror symmetry will be an isomorphism between this structure and a similar structure on $\mathcal{K}_{\mathbb{C}}\left(X_{\mathcal{J}}\right) / \operatorname{Aut}\left(X_{\mathcal{J}}\right)$.

In fact, the conformal field theory naturally encodes a variation of Hodge structure on $\mathcal{K}_{\mathbb{C}}\left(X_{\mathcal{J}}\right) / \operatorname{Aut}\left(X_{\mathcal{J}}\right)$. To describe this mathematically, we must choose a framing, which is a choice of cone

$$
\sigma=\mathbb{R}_{+} e^{1}+\cdots+\mathbb{R}_{+} e^{r} \subset H^{2}(X, \mathbb{R})
$$

which is generated by a basis $e^{1}, \ldots, e^{r}$ of $H^{2}(X, \mathbb{Z}) /$ torsion and whose interior is contained in the Kähler cone of $X$. The complexified Kähler part of the semiclassical moduli space then contains as an open subset the space

$$
\mathcal{M}_{A}(\sigma):=\left(H^{2}(X, \mathbb{R})+i \sigma\right) / H^{2}(X, \mathbb{Z}),
$$

elements of which can be expanded in the form $\sum\left(\frac{1}{2 \pi i} \log q_{j}\right) e^{j}$, leading to the alternate description

$$
\mathcal{M}_{A}(\sigma)=\left\{\left(q_{1}, \ldots, q_{r}\right)|0<| q_{j} \mid<1\right\} .
$$

The desired variation of Hodge structure will be defined on a partial compactification of this space, namely

$$
\overline{\mathcal{M}}_{A}(\sigma):=\left\{\left(q_{1}, \ldots, q_{r}\right)|0 \leq| q_{j} \mid<1\right\},
$$

\footnotetext{
${ }^{14}$ Note that $\mathcal{F}^{n}$ appears directly in the conformal field theory, and $\mathcal{F}^{n-1} / \mathcal{F}^{n}$ appears as a class of marginal operators in the conformal field theory. Thus, the conformal field theory contains at least as much of the Hodge-theoretic data as is described by the smallest sub-Hodge structure containing $\mathcal{F}^{n-1}$, and quite possibly more.
} 
which has a distinguished boundary point $\overrightarrow{0}=(0, \ldots, 0)$.

The ingredients we need to define the variation of Hodge structure are the fundamental Gromov-Witten invariants ${ }^{\mathrm{T}}$ of $X$, which are trilinear maps

$$
\Phi_{\eta}^{0}: H^{*}(X, \mathbb{Q}) \oplus H^{*}(X, \mathbb{Q}) \oplus H^{*}(X, \mathbb{Q}) \rightarrow \mathbb{Q} .
$$

Heuristically, when $A, B$ and $C$ are integral classes, $\Phi_{\eta}^{0}(A, B, C)$ should

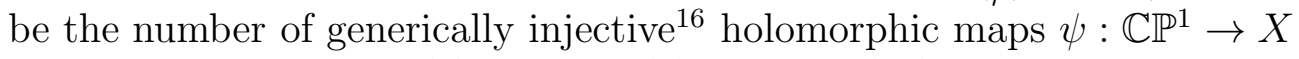
in class $\eta$, such that $\psi(0) \in Z_{A}, \psi(1) \in Z_{B}, \psi(\infty) \in Z_{C}$ for appropriate cycles $Z_{A}, Z_{B}, Z_{C}$ Poincaré dual to the classes $A, B, C$, respectively. (The invariants vanish unless $\operatorname{deg} A+\operatorname{deg} B+\operatorname{deg} C=2 n$.) From these invariants we can define the Gromov-Witten maps $\Gamma_{\eta}: H^{k}(X) \rightarrow H^{k+2}(X)$ by requiring that

$$
\left.\Gamma_{\eta}(A) \cdot B\right|_{[X]}=\frac{\Phi_{\eta}^{0}(A, B, C)}{\eta \cdot C}
$$

for $B \in H^{2 n-k-2}(X), C \in H^{2}(X)$. (This is independent of the choice of C.)

These invariants are usually assembled into the "quantum cohomology ring" of $X$, but here we present this structure in the equivalent form of a variation of Hodge structure over $\overline{\mathcal{M}}_{A}(\sigma)$ degenerating along the boundary. To do so, we define a holomorphic vector bundle $\mathcal{E}:=$ $\left(\bigoplus H^{\ell, \ell}(X)\right) \otimes \mathcal{O}_{\overline{\mathcal{M}}_{A}(\sigma)}$, and a flat with regular singular points along the boundary $\mathcal{B}=\overline{\mathcal{M}}_{A}(\sigma)-\mathcal{M}_{A}(\sigma)$ by the formulat

$$
\nabla:=\frac{1}{2 \pi i}\left(\sum_{j=1}^{r} d \log q_{j} \otimes \operatorname{ad}\left(e^{j}\right)+\sum_{0 \neq \eta \in H_{2}(X, \mathbb{Z})} d \log \left(\frac{1}{1-q^{\eta}}\right) \otimes \Gamma_{\eta}\right)
$$

where $q^{\eta}=\prod q_{j}^{e^{j}(\eta)}$, and where $\operatorname{ad}\left(e^{j}\right): H^{k}(X) \rightarrow H^{k+2}(X)$ is the adjoint map of the cup product pairing, defined by $\operatorname{ad}\left(e^{j}\right)(A)=e^{j} \cup A$. We also define a "Hodge filtration"

$$
\mathcal{E}^{p}:=\left(\bigoplus_{0 \leq \ell \leq n-p} H^{\ell, \ell}(X)\right) \otimes \mathcal{O}_{\overline{\mathcal{M}}_{A}(\sigma)},
$$

which satisfies $\nabla\left(\mathcal{E}^{p}\right) \subset \Omega \frac{1}{\mathcal{M}}(\log \mathcal{B}) \otimes \mathcal{E}^{p-1}$. This describes a structure we call the framed A-variation of Hodge structure with framing $\sigma$. To be

\footnotetext{
${ }^{15}$ These can be defined using techniques from symplectic geometry 44, 36, 50, or from algebraic geometry [32, 31, 15, 14, 13, 34.

${ }^{16}$ We have built the "multiple cover formula" 3, 35, 54 into our definitions.

${ }^{17}$ The flatness of this connection is equivalent to the associativity of the product in quantum cohomology.

${ }^{18} \mathrm{I}$ am indebted to P. Deligne for advice [20] which led to this form of the formula.
} 
a bit more precise, we should refer to this as a "formally degenerating variation of Hodge structure," since the series used to define $\nabla$ is only formal. (More details about such structures can be found in 43]; cf. also 200.) There are also some subtleties about passing from a local system of complex vector spaces to a local system of $\mathbb{Z}$-modules which we shall discuss in section 5 below.

The residues of $\nabla$ along the boundary components $q_{j}=0$ are the adjoint maps $\operatorname{ad}\left(e^{j}\right)$; the corresponding monodromy weight filtration at $\overrightarrow{0}$ is simply

$$
H_{0,0}(X) \subseteq H_{0,0}(X) \oplus H_{1,1}(X) \subseteq \cdots \subseteq\left(H_{0,0}(X) \oplus \cdots \oplus H_{n, n}(X)\right) .
$$

Under mirror symmetry, this maps to the geometric monodromy weight filtration at an appropriate "large complex structure limit" point in $\overline{\mathcal{M}}_{c x}$ (see 41] and references therein). Note that the class of the 0-cycle is the monodromy-invariant class in $H_{\text {even }}(X)$; thus, its mirror $n$-cycle will be the monodromy-invariant class in $H_{n}(Y)$.

Although the choice of a "framing" may look unnatural, the relationship between different choices of framing is completely understood 38 (modulo a conjecture about the action of the automorphism group on the Kähler cone). Varying the framing corresponds to varying which boundary point in the moduli space one is looking at, possibly after blowing up the original boundary of the moduli space in order to find an appropriate compactification containing the desired boundary point.

We finally come to the definition which contains our precise Hodgetheoretic mirror prediction from physics.

Definition 3. Let $X$ and $Y$ be Calabi-Yau manifolds with $h^{2,0}(X)=$ $h^{2,0}(Y)=0$. The pair $(X, Y)$ passes the Hodge-theoretic mirror test if there exists a partial compactification $\overline{\mathcal{M}}_{c x}(Y)$ of the complex structure moduli space of $Y$, a neighborhood $U \subset \overline{\mathcal{M}}_{c x}(Y)$ of a boundary point $P$ of $\overline{\mathcal{M}}_{c x}(Y)$, a framing $\sigma$ for $H^{2}(X)$, and a "mirror map" $\mu: U \rightarrow \mathcal{M}_{A}(\sigma)$ mapping $P$ to $\overrightarrow{0}$ such that $\mu^{*}$ induces an isomorphism between $\mathcal{E}^{n-1}$ and $\mathcal{F}^{n-1}$ which extends to an isomorphism between sub-variations of Hodge structure of the A-variation of Hodge structure with framing $\sigma$, and the geometric formally degenerating variation of Hodge structure at $P$.

The restriction to a sub-variation of Hodge structure (which occurs only when the dimension of the Calabi-Yau manifold is greater than three) seems to be necessary in order to get an integer structure on the local system compatible with the complex variation of Hodge structure. (We will return to this issue in section 5.) It seems likely that this is related to the need to pass to "string-theoretic Hodge numbers," which 
may actually be measuring the Hodge numbers of the appropriate subHodge structures.

The property described in the Hodge-theoretic mirror test can be recast in terms of using the limiting variation of Hodge structure on $Y$ to make predictions about enumerative geometry of holomorphic rational curves on $X$. In this sense, there is a great deal of evidence in particular cases (see 41, 25] and the references therein). There are also some specific connections which have been found between the variations of Hodge structure associated to mirror pairs of theories [44], as well as a recent theorem [23] which proves that the expected enumerative properties hold for an important class of Calabi-Yau manifolds.

Note that if $(X, Y)$ passes the Hodge-theoretic mirror test in both directions, then it passes the topological mirror test (essentially by definition, since the dimensions of the moduli spaces are given by the Hodge numbers $h^{1,1}$ and $\left.h^{n-1,1}\right)$.

\section{Geometric MirRor PAIRS}

We now wish to translate the Strominger-Yau-Zaslow analysis into a definition of geometric mirror pairs $(X, Y)$, which we formulate in arbitrary dimension. (As mentioned earlier, the arguments of 53 cannot be applied to conclude that all mirror pairs arise in this way, but it seems reasonable to suppose that a T-duality argument-applied to conformal field theories only - would continue to hold.) The most straightforward such definition would say that $X$ is the compactification of the complexified moduli space of special Lagrangian $n$-tori on $Y$. However, as indicated by our conjectures of section 2, at present we do not have adequate technical control over the compactification to see that it is a Calabi-Yau manifold. So we make instead an indirect definition, motivated by the following observation: if we had such a compactified moduli space $X$, then for generic $x \in X$ there would be a corresponding special Lagrangian $n$-torus $T_{x} \subset Y$, and we could define an incidence correspondence

$$
Z=\text { closure of }\left\{(x, y) \in X \times Y \mid y \in T_{x}\right\} .
$$

By definition, the projection $Z \rightarrow X$ would have special Lagrangian $n$ tori as generic fibers. As we saw earlier, from the analysis of [53 it is expected that generic fibers of the other projection $Z \rightarrow Y$ will also be special Lagrangian $n$-tori. Furthermore, we should expect that as we vary the metrics on $X$ and on $Y$, the fibrations by special Lagrangian $n$-tori can be deformed along with the metrics. (In fact, it is these dependencies on parameters which should lead to a "mirror map" between moduli spaces.) Thus, we will formulate our definition using a family of correspondences depending on $t \in U$ for some (unspecified) parameter space $U$. 
Definition 4. A pair of Calabi-Yau manifolds $(X, Y)$ is a geometric mirror pair if there is a parameter space $U$ such that for each $t \in U$ there exist

1. a correspondence $Z_{t} \subset(X \times Y)$ which is the closure of a submanifold of dimension $3 n$,

2. maps $\tau_{t}: X \rightarrow Z_{t}$ and $\widetilde{\tau}_{t}: Y \rightarrow Z_{t}$ which serve as sections for the projection maps $Z_{t} \rightarrow X$ and $Z_{t} \rightarrow Y$, respectively,

3. a Ricci-flat metric $g_{i j}(t)$ on $X$ with respect to which generic fibers of the projection map $Z_{t} \rightarrow Y$ are special Lagrangian $n$-tori, and

4. a Ricci-flat metric $\widetilde{g}_{i j}(t)$ on $Y$ with respect to which generic fibers of the projection map $Z_{t} \rightarrow X$ are special Lagrangian $n$-tori.

Moreover, for generic $z \in Z_{t}$, the fibers through $z$ of the two projection maps must be canonically dual as tori (with origins specified by $\tau_{t}$ and $\widetilde{\tau}_{t}$ ).

In a somewhat stronger form of the definition, we might require that $U$ be sufficiently large so that the images of the natural maps $U \rightarrow \mathcal{M}_{R i c}(X)$ and $U \rightarrow \mathcal{M}_{R i c}(Y)$ to the moduli spaces of Ricci-flat metrics on $X$ and on $Y$ are open subsets of the respective moduli spaces. It is too much to hope that these maps would be surjective. The best picture we could hope for, in fact, would be a diagram of the form

$$
\mathcal{M}_{\text {Ric }}(X) \supseteq U_{X} \stackrel{\pi_{X}}{\longleftarrow} U \stackrel{\pi_{Y}}{\longrightarrow} U_{Y} \subseteq \mathcal{M}_{\text {Ric }}(Y)
$$

in which $U_{X} \subseteq \mathcal{M}_{R i c}(X)$ and $U_{Y} \subseteq \mathcal{M}_{R i c}(Y)$ are open subsets (near certain boundary points in a compactification and contained within the set of metrics for which the semiclassical approximation is valid). The fibers of $\pi_{X}$ will have dimension $h^{1,1}(X)$, and if the induced map is the mirror map each fiber of $\pi_{X}$ must essentially be the set of $B$-fields on $X$, i.e., it must be a deformation of the real torus $H^{2}(X, \mathbb{R} / \mathbb{Z})$. This is compatible with the approximate formula ${ }^{\top}$ in 53 for a family of metrics on $Y$, produced by varying the $B$-field on $X$.

We expect that geometric mirror symmetry will be related to the earlier mathematical mirror symmetry properties in the following way.

Conjecture 3. If $(X, Y)$ is a geometric mirror pair, then the parameter space $U$ and the data in the definition of the geometric mirror pair can be chosen so that

1. $(X, Y)$ passes the topological mirror test?,

2. $\pi_{X}: U \rightarrow \mathcal{M}_{\text {Ric }}(X)$ lifts to a generically finite map $\widetilde{\pi}_{X}: U \rightarrow \widetilde{U}_{X} \subseteq$ $\mathcal{M}_{C F T}^{s c}(X)$,

\footnotetext{
${ }^{19}$ The "tree-level" formula given in 53] is subject to unspecified instanton corrections.

${ }^{20}$ Part (11) is a consequence of part (A) if $h^{2,0}(X)=h^{2,0}(Y)=0$.
} 
3. $\pi_{Y}: U \rightarrow \mathcal{M}_{R i c}(Y)$ lifts to a generically finite map $\widetilde{\pi}_{Y}: U \rightarrow \widetilde{U}_{Y} \subseteq$ $\mathcal{M}_{C F T}^{s c}(Y)$, and

4. if $h^{2,0}(X)=h^{2,0}(Y)=0$, then there are boundary points $P \in$ $\overline{\mathcal{M}}_{c x}(Y), P^{\prime} \in \overline{\mathcal{M}}_{c x}(X)$ and framings $\sigma$ of $H^{2}(X)$ and $\sigma^{\prime}$ of $H^{2}(Y)$ with partial compactifications $\overline{\widetilde{U}}_{X} \subset \mathcal{M}_{A}(\sigma) \times \overline{\mathcal{M}}_{c x}(X)$ and $\overline{\widetilde{U}}_{Y} \subset$ $\overline{\mathcal{M}}_{c x}(Y) \times \mathcal{M}_{A}\left(\sigma^{\prime}\right)$ such that the composite map $\left(\widetilde{\pi}_{X}\right)_{*}\left(\widetilde{\pi}_{Y}\right)^{*}$ extends to a map $\mu^{-1} \times \mu^{\prime}$ which consists of mirror maps in both directions (in the sense of definition (3). In particular, $(X, Y)$ passes the Hodgetheoretic mirror test.

Even in the case that $h^{2,0}(X) \neq 0$, there is an induced map $\left(\widetilde{\pi}_{X}\right)_{*}\left(\widetilde{\pi}_{Y}\right)^{*}$ which should coincide with the mirror map between the moduli spaces.

If $X$ has several birational models $X^{(j)}$, then all of the semiclassical moduli spaces $\mathcal{M}_{C F T}^{s c}\left(X^{(j)}\right)$ give rise to a common conformal field theory moduli space (see [1], or for a more mathematical account, [39]). If we follow a path between the large radius limit points of two of these models, and reinterpret that path in the mirror moduli space, we find a path which leads from one large complex structure limit point of $\mathcal{M}_{c x}(Y)$ to another. On the other hand, the calculation of [2] shows that the homology class of the torus ${ }^{27}$ in a special Lagrangian $T^{n}$-fibration does not change when we move from one of these regions of $\mathcal{M}_{c x}(Y)$ to another. Thus, the moduli space of special Lagrangian $T^{n}$ 's themselves must change as we move from region to region. It will be interesting to investigate precisely how this change comes about.

\section{Mirror COHOMOLOGY AND THE WEIGHT FILTRATION}

The "duality" transformation which links the two members $X$ and $Y$ of a geometric mirror pair does not induce any obvious relationship between $H^{1,1}(X)$ and $H^{n-1,1}(Y)$, so it may be difficult to imagine how the topological mirror test can be passed by a geometric mirror pair. However, at least for a restricted class of topological cycles, such a relationship can be found, as part of a more general relationship between certain subspaces of $H_{\text {even }}(X)$ and $H_{n}(Y)$.

Fix a special Lagrangian $T^{n}$-fibration on $Y$ with a special Lagrangian section, and consider $n$-cycles $W \subset Y$ with the property that $W$ is the closure of a submanifold $W_{0}$ whose intersection with each nonsingular $T^{n}$ in the fibration is either empty, or a sub-torus of dimension $n-k$ (for some fixed integer $k \leq n$ ). That is, we assume that $W$ can be generically described as a $T^{n-k}$-bundle over a $k$-manifold, with the $T^{n-k}$ 's linearly embedded in fibers of the given $T^{n}$-fibration. We call such $n$-cycles pure.

\footnotetext{
${ }^{21}$ Recall that this is the monodromy-invariant cycle.
} 
For any pure $n$-cycle $W \subset Y$, there is a $T$-dual cycle $W^{\vee 2} \subset X(=$ $\left.\overline{\mathcal{M}}_{D}\left(T^{n}, Y\right)\right)$ defined as the closure of an $n$-manifold $W_{0}^{\vee}$ satisfying

$$
W_{0}^{\vee} \cap\left(T^{n}\right)^{*}= \begin{cases}\text { the annihilator of } W \cap T^{n} \text { in }\left(T^{n}\right)^{*} & \text { if } W \cap T^{n} \neq \emptyset \\ \emptyset & \text { otherwise }\end{cases}
$$

for all smooth fibers $\left(T^{n}\right)^{*}$ in the dual fibration. Since the annihilator of an $(n-k)$-torus is a $k$-torus, we see that $W^{\vee}$ is generically described as a $T^{k}$-bundle over a $k$-manifold, and so it defines a class in $H_{2 k}(X)$. This is our relationship between the space of pure $n$-cycles on $Y$, and the even homology on $X$.

Taking the T-duality statements from physics very literally, we are led to the speculation that pure special Lagrangian $n$-cycles have as their T-duals certain algebraic cycles on $X$; moreover, the moduli spaces containing corresponding cycles should be isomorphic. ${ }^{23}$ (Roughly speaking, the $T^{k}$-fibration on the corresponding algebraic $k$-cycle should be given by holding the norms of some system of complex coordinates on the $k$ cycle fixed, while varying their arguments.) The simplest cases of this statement we have already encountered in the Strominger-Yau-Zaslow discussion: the special Lagrangian $n$-cycles which consist of a single fiber (i.e., $k=0$ ) are T-dual to the 0 -cycles of length one on $X$, while a special Lagrangian $n$-cycle which is the zero-section of the fibration (i.e., $k=n$ ) is T-dual to the $2 n$-cycle in the fundamental class. This new construction should extend that correspondence between cycles to a broader class (albeit still a somewhat narrow one, since pure cycles are quite special).

In fact, the correspondence should be even broader. If we begin with an arbitrary irreducible special Lagrangian $n$-cycle $W$ on $Y$ whose image in $\mathcal{M}_{s L}\left(T^{n}, Y\right)$ has dimension $k$, then $W$ can be generically described as a bundle of $(n-k)$-manifolds over the image $k$-manifold. The T-dual of such a cycle should be a coherent sheaf $\mathcal{E}$ on $X$ whose support $Z$ is a complex submanifold of dimension $k$ whose image in $\mathcal{M}_{s L}\left(T^{n}, Y\right)$ is that same $k$-manifold. Thus, to the homology class of $W$ in $H_{n}(Y)$ we associate the total homology class in $H_{\text {even }}(X)$ of the corresponding

\footnotetext{
${ }^{22}$ In physics, when a T-duality transformation is applied to a real torus, a D-brane supported on a sub-torus is mapped to a D-brane supported on the "dual" sub-torus (of complementary dimension); this can be mathematically identified as the annihilator. Here, we apply this principle to a family of sub-tori within a family of tori.

${ }^{23}$ As the referee has pointed out, our "purity" condition is probably too strong to be preserved under deformation, but one can hope that all nearby deformations of a (pure) special Lagrangian $n$-cycle are reflected in deformations of the corresponding algebraic cycle.
} 
coherent sheaf Note that since the support has complex dimension $k$, this total homology class lies in $H_{0}(X) \oplus H_{2}(X) \oplus \cdots \oplus H_{2 k}(X)$.

The homology class of the generic fiber of $W$ within $T^{n}$ should determine the sub-tori whose T-duals would sweep out $Z$; when that homology class is $r$ times a primitive class, the corresponding coherent sheaf should have generic rank $r$ along $Z$. For example, a multi-section of the special Lagrangian $T^{n}$ fibration which meets the fiber $r$ times should correspond to a coherent sheaf whose support is all of $X$ and whose rank is $r$.

We have thus found a mapping from the subspace $H_{n}^{s L}(Y)$ of $n$-cycles with a special Lagrangian representative, to the subspace $H_{\text {even }}^{\text {alg }}(Y)$ of homology classes of algebraic cycles (and coherent sheaves). If we consider the Leray filtration on special Lagrangian $n$-cycles on $Y$

$$
\mathbb{S}_{k}:=\left\{W \in H_{n}^{s L}(Y) \mid \operatorname{dim}(\text { image } W) \leq k\right\},
$$

then this will map to

$$
H_{0}^{a l g}(X) \oplus H_{2}^{a l g}(X) \oplus \cdots \oplus H_{2 k}^{a l g}(X)
$$

(and the pure $n$-cycles on $Y$ will map to homology classes of algebraic cycles on $Y$ ). But this latter filtration on $H_{\text {even }}(X)$ is precisely the monodromy weight filtration of the $A$-variation of Hodge structures on $X$, which should be mirror to the geometric monodromy weight filtration on $Y$ We are thus led to the following refinement of conjecture 3 .

Conjecture 4. If $(X, Y)$ is a geometric mirror pair then there exists a large complex structure limit point $P \in \overline{\mathcal{M}}_{c x}(Y)$ corresponding to the mirror partner $X$, and a sub-variation of the geometric variation of Hodge structure defined on $H_{n}^{s L}(Y)^{*}$ whose monodromy weight filtration at $P$ coincides with the Leray filtration for the special Lagrangian $T^{n}$-fibration on $Y$. Moreover, under the isomorphism of conjecture \$, this maps to the sub-variation of the A-variation of Hodge structure defined on $H_{\text {even }}^{\text {alg }}(X)^{*}$.

The difficulty in putting an integer structure on the $A$-variation of Hodge structure stems from the fact that $H^{p, p}(X)$ will in general not be generated by its intersection with $H^{2 p}(X, \mathbb{Z})$. However, the algebraic cohomology $H_{\text {even }}^{\text {alg }}(X)^{*}$ does not suffer from this problem: its graded pieces are generated by integer $(p, p)$-classes. If conjecture 4 holds, it explains why there is a corresponding sub-variation of the geometric variation of Hodge structure on $Y$, also defined over the integers. We would thus get corresponding local systems over $\mathbb{Z}$ in addition to the isomorphisms of complex variations of Hodge structure.

\footnotetext{
${ }^{24}$ It appears from both the K3 case discussed in the next section, and the analysis of 24. that the correct total homology class to use is the Poincaré dual of $\operatorname{ch}(\mathcal{E}) \sqrt{\operatorname{td} Y}$.

${ }^{25}$ This property of the mapping of D-branes has also been observed by Ooguri, $\mathrm{Oz}$ and Yin 48 .
} 


\section{Geometric MirRor Symmetry FOR K3 SURFACES}

The special Lagrangian submanifolds of a K3 surface can be studied directly, thanks to the following fact due to Harvey and Lawson [29]: given a Ricci-flat metric on a K3 surface $Y$ and a special Lagrangian submanifold $M$, there exists a complex structure on $Y$ with respect to which the metric is Kähler, such that $M$ is a complex submanifold of $Y$. This allows us to immediately translate the theory of special Lagrangian $T^{2}$-fibrations on $Y$ to the standard theory of elliptic fibrations. In this section, we will discuss geometric mirror symmetry for K3 surfaces in some detail. (Some aspects of this case have also been worked out by Gross and Wilson [28], who went on to study geometric mirror symmetry for the Voisin-Borcea threefolds of the form $\left(\mathrm{K} 3 \times T^{2}\right) / \mathbb{Z}_{2}$.)

If we fix a cohomology class $\mu \in H^{2}(Y, \mathbb{Z})$ which is primitive (i.e., $\frac{1}{n} \mu \notin H^{2}(Y, \mathbb{Z})$ for $\left.1<n \in \mathbb{Z}\right)$ and satisfies $\mu \cdot \mu=0$, then for any Ricciflat metric we can find a compatible complex structure for which $\mu$ has type $(1,1)$ and $\kappa \cdot \mu>0$ ( $\kappa$ being the Kähler form). The class $\mu$ is then represented by a complex curve, which moves in a one-parameter family, defining the structure of an elliptic fibration. Thus, elliptic fibrations of this sort exist for every Ricci-flat metric on a K3 surface. ${ }^{20}$

Our conjecture 1 is easy to verify in this case: as is well-known, the base of the elliptic fibration on a K3 surface can be completed to a 2-sphere, and the resulting map from $\mathrm{K} 3$ to $S^{2}$ is proper. In fact, the possible singular fibers are known very explicitly in this case [30.

To study conjecture 2, we need to understand the structure of the "complexified" moduli space $\mathcal{M}_{D}\left(T^{2}, Y\right)$. Since a flat $U(1)$-bundle on an elliptic curve is equivalent to a holomorphic line bundle of degree zero, each point in $\mathcal{M}_{D}\left(T^{2}, Y\right)$ has a natural interpretation as such a bundle on some particular fiber of the elliptic fibration. Extending that bundle by zero, we can regard it as a sheaf $\mathcal{L}$ on $Y$, with $\operatorname{supp}(\mathcal{L})=\operatorname{image}(f)$. We thus identify $\mathcal{M}_{D}\left(T^{2}, Y\right)$ as a moduli spaces of such sheaves.

Let us briefly recall the facts about the moduli spaces of simple sheaves on K3 surfaces, as worked out by Mukai [46, 47. First, Mukai showed that for any simple sheaf $\mathcal{E}$ on $Y$, i.e., one without any non-constant endomorphisms, the moduli space $M_{\text {simple }}$ is smooth at $[\mathcal{E}]$ of dimension $\operatorname{dim} \operatorname{Ext}^{1}(\mathcal{E}, \mathcal{E})=2-\chi(\mathcal{E}, \mathcal{E})$. (The " 2 " in the formula arises from the spaces $\operatorname{Hom}(\mathcal{E}, \mathcal{E})$ and $\operatorname{Ext}^{2}(\mathcal{E}, \mathcal{E})$, each of which has dimension one, due

\footnotetext{
${ }^{26}$ They even exist - although possibly in degenerate form-for the "orbifold" metrics which occur at certain limit points of the moduli space: at those points, $\kappa$ is only required to be semi-positive, but by the index theorem $\kappa^{\perp}$ cannot contain an isotropic vector such as $\mu$, so it is still possible to choose a complex structure such that $\kappa \cdot \mu>0$.
} 
to the constant endomorphisms in the first case, and their Kodaira-Serre duals in the second case.)

Second, Mukai introduced an intersection pairing on $H^{e v}(Y)=H^{0}(Y) \oplus$ $H^{2}(Y) \oplus H^{4}(Y)$ defined by

$$
(\alpha, \beta, \gamma) \cdot\left(\alpha^{\prime}, \beta^{\prime}, \gamma^{\prime}\right)=\left.\left(\beta \cdot \beta^{\prime}-\alpha \cdot \gamma^{\prime}-\gamma \cdot \alpha^{\prime}\right)\right|_{[Y]},
$$

and a slight modification of the usual Chern character $\operatorname{ch}(\mathcal{E})$, defined by

$$
v(\mathcal{E})=\operatorname{ch}(\mathcal{E}) \sqrt{\operatorname{td}(Y)}=\left(\left(\operatorname{rank}(\mathcal{E}), c_{1}(\mathcal{E}), \operatorname{rank}(\mathcal{E})+\frac{1}{2}\left(c_{1}(\mathcal{E})^{2}-2 c_{2}(\mathcal{E})\right),\right.\right.
$$

so that the Riemann-Roch theorem reads

$$
\chi(\mathcal{E}, \mathcal{F})=v(\mathcal{E}) \cdot v(\mathcal{F}) .
$$

In particular, the moduli space $\mathcal{M}_{\text {simple }}(v)$ of simple sheaves with $v(\mathcal{E})=$ $v$ has dimension

$$
\operatorname{dim} \mathcal{M}_{\text {simple }}(v)=2-\chi(\mathcal{E}, \mathcal{E})=2-v \cdot v .
$$

In the case of moduli spaces $\mathcal{M}_{\text {simple }}(v)$ of dimension two, Mukai went on to show that whenever the space is compact, it must be a K3 surface.

The sheaves $\mathcal{L}$ with support on a curve from our elliptic fibration will have Mukai class $v(\mathcal{L})=(0, \mu, 0)$ for which $v(\mathcal{L}) \cdot v(\mathcal{L})=\mu \cdot \mu=0$, so the moduli space has dimension two. That is, our moduli space $\mathcal{M}_{D}\left(T^{2}, Y\right)$ is contained in $\mathcal{M}_{\text {simple }}(0, \mu, 0)$ as an open subset. Our second conjecture will follow if we can show that this latter space is compact, or at least admits a natural compactification. Whether this is true or not could in principle depend on the choice of Ricci-flat metric on $Y$. If we restrict to metrics with the property that $Y$ is algebraic when given the compatible complex structure for which $\mu$ defines an elliptic fibration (this is a dense set within the full moduli space), then techniques of algebraic geometry can be applied to this problem. General results of Simpson [51] imply that on an algebraic K3 surface, the set of semistable sheaves with a fixed Mukai vector $v$ forms a projective variety. This applies to our situation with $v=(0, \mu, 0)$, and provides the desired compactification. It is to be hoped that compactifications such as this exist even for non-algebraic K3 surfaces.

The Mukai class $v=(0, \mu, 0)$ should now be mapped under mirror symmetry to the class of a zero-cycle, or the corresponding sheaf $\mathcal{O}_{P}$; that Mukai class is $(0,0,1)$. In fact, the mirror map known in physics [4] does precisely that: given any primitive isotropic vector $v$ in $H^{e v}(Y)$, there is a mirror map which takes it to the vector $(0,0,1)$. Moreover, it is easy to calculate how this mirror map affects complex structures, by specifying how it affects Hodge structures: if we put a Hodge structure on $H^{e v}(Y)$ in which $H^{0}$ and $H^{4}$ have been specified as type $(1,1)$, then 
the corresponding Hodge structure at the mirror image point has $v^{\perp} / v$ as its $H^{2}$.

This is precisely the relationship between Hodge structures on $Y$ and on $\mathcal{M}_{\text {simple }}(v)$ which was found by Mukai [47]! We can thus identify geometric mirror symmetry for K3 surfaces (which associates the moduli spaces of zero-cycles and special Lagrangian $T^{2}$ 's) with the mirror symmetry previously found in physics. It is amusing to note that in establishing this relationship, Mukai used elliptic fibrations and bundles on them in a crucial way.

As suggested in the previous section, such a mirror transformation should act on the totality of special Lagrangian 2-cycles. In fact, it is known that for at least some K3 surfaces, there is a Fourier-Mukai transform which associates sheaves on $\mathcal{M}_{\text {simple }}(v)$ to sheaves on $Y$ [7]. The map between their homology classes is precisely the mirror map. 27 Thus, proving that there exists such a Fourier-Mukai transform for arbitrary K3 surfaces (even non-algebraic ones) would establish a version of conjecture t in this case.

Acknowledgments. It is a pleasure to thank Robbert Dijkgraaf, Ron Donagi, Brian Greene, Mark Gross, Paul Horja, Sheldon Katz, Greg Moore, Ronen Plesser, Yiannis Vlassopoulos, Pelham Wilson, Edward Witten, and especially Robert Bryant and Andy Strominger for useful discussions; I also thank Strominger for communicating the results of 553 prior to publication, and the referee for useful remarks on the first version. I am grateful to the Rutgers physics department for hospitality and support during the early stages of this work, to the organizers of the European Algebraic Geometry Conference at the University of Warwick where this work was first presented, and to the Aspen Center for Physics where the writing was completed. This research was partially supported by the National Science Foundation under grant DMS-9401447.

\section{REFERENCES}

[1] P. S. Aspinwall, B. R. Greene, and D. R. Morrison, Calabi-Yau moduli space, mirror manifolds and spacetime topology change in string theory, Nuclear Phys. B 416 (1994), 414-480, hep-th/9309097.

[2] _ Measuring small distances in $N=2$ sigma models, Nuclear Phys. B 420 (1994), 184-242, hep-th/9311042.

[3] P. S. Aspinwall and D. R. Morrison, Topological field theory and rational curves, Comm. Math. Phys. 151 (1993), 245-262, hep-th/9110048.

\footnotetext{
${ }^{27}$ One example of this is given by a special Lagrangian section of the $T^{2}$-fibration, which will map to the class $(1,0,1)$ which is the Mukai vector of the fundamental cycle (i.e., of the structure sheaf) on the mirror.
} 
[4] _ String theory on K3 surfaces, Mirror Symmetry II (B. Greene and S.-T. Yau, eds.), International Press, Cambridge, 1997, pp. 703-716, hep-th/9404151.

[5] Chiral rings do not suffice: $N=(2,2)$ theories with nonzero fundamental group, Phys. Lett. B 334 (1994), 79-86, hep-th/9406032.

[6] , U-duality and integral structures, Phys. Lett. B 355 (1995), 141-149, hep-th/9505025.

[7] C. Bartocci, U. Bruzzo, and D. Hernández Ruipérez, A Fourier-Mukai transform for stable bundles on K3 surfaces, J. Reine Angew. Math. 486 (1997), 1-16, alg-geom/9405006.

[8] V. V. Batyrev, Dual polyhedra and mirror symmetry for Calabi-Yau hypersurfaces in toric varieties, J. Algebraic Geom. 3 (1994), 493-535, alg-geom/9310003.

[9] V. V. Batyrev and L. A. Borisov, Dual cones and mirror symmetry for generalized Calabi-Yau manifolds, Mirror Symmetry II (B. Greene and S.-T. Yau, eds.), International Press, Cambridge, 1997, pp. 71-86, alg-geom/9402002.

[10] _ Mirror duality and string-theoretic Hodge numbers, Invent. Math. 126 (1996), 183-203, alg-geom/9509009.

[11] V. V. Batyrev and D. I. Dais, Strong McKay correspondence, stringtheoretic Hodge numbers and mirror symmetry, Topology, 35 (1996), 901-929, alg-geom/9410001.

[12] K. Becker, M. Becker, and A. Strominger, Fivebranes, membranes and non-perturbative string theory, Nuclear Phys. B 456 (1995), 130-152, hep-th/9507158.

[13] K. Behrend, Gromov-Witten invariants in algebraic geometry, Invent. Math. 127 (1997), 601-617, alg-geom/9601011.

[14] K. Behrend and B. Fantechi, The intrinsic normal cone, Invent. Math. 128 (1997), 45-88, alg-geom/9601010.

[15] K. Behrend and Yu. Manin, Stacks of stable maps and Gromov-Witten invariants, Duke Math. J. 85 (1996), 1-60, alg-geom/9506023.

[16] L. Borisov, Towards the mirror symmetry for Calabi-Yau complete intersections in toric Fano varieties, alg-geom/9310001.

[17] R. L. Bryant, Submanifolds and special structures on the octonians, J. Differential Geom. 17 (1982), 185-232.

[18] _ Minimal Lagrangian submanifolds of Kähler-Einstein manifolds, Differential Geometry and Differential Equations, Shanghai 1985, Lecture Notes in Math., vol. 1255, 1985, pp. 1-12.

[19] P. Candelas, M. Lynker, and R. Schimmrigk, Calabi-Yau manifolds in weighted $\mathbb{P}_{4}$, Nuclear Phys. B 341 (1990), 383-402.

[20] P. Deligne, Local behavior of Hodge structures at infinity, Mirror Symmetry II (B. Greene and S.-T. Yau, eds.), International Press, Cambridge, 1997, pp. 683700.

[21] L. J. Dixon, Some world-sheet properties of superstring compactifications, on orbifolds and otherwise, Superstrings, Unified Theories, and Cosmology 1987 (G. Furlan et al., eds.), World Scientific, 1988, pp. 67-126.

[22] R. Donagi and E. Markman, Cubics, integrable systems, and Calabi-Yau threefolds, Proc. of the Hirzebruch 65 Conference on Algebraic Geometry (M. Teicher, ed.), Israel Math. Conf. Proc., vol. 9, Bar-Ilan University, 1996, pp. 199-221, alg-geom/9408004.

[23] A. B. Givental, Equivariant Gromov-Witten invariants, Internat. Math. Res. Notices (1996), 613-663, alg-geom/9603021. 
[24] M. Green, J. A. Harvey, and G. Moore, I-brane inflow and anomalous couplings on D-branes, Class. Quant. Grav. 14 (1997), 47-52, hep-th/9605033.

[25] B. R. Greene, D. R. Morrison, and M. R. Plesser, Mirror manifolds in higher dimension, Comm. Math. Phys. 173 (1995), 559-598, hep-th/9402119.

[26] B. R. Greene, D. R. Morrison, and A. Strominger, Black hole condensation and the unification of string vacua, Nuclear Phys. B 451 (1995), 109-120, hep-th/9504145.

[27] B. R. Greene and M. R. Plesser, Duality in Calabi-Yau moduli space, Nuclear Phys. B 338 (1990), 15-37.

[28] M. Gross and P. M. H. Wilson, Mirror symmetry via 3-tori for a class of CalabiYau threefolds, Math. Ann., to appear, alg-geom/9608004.

[29] R. Harvey and H. B. Lawson, Jr., Calibrated geometries, Acta Math. 148 (1982), $47-157$.

[30] K. Kodaira, On compact analytic surfaces, II, III, Ann. of Math. (2) 77 (1963), 563-626; 78 (1963), 1-40.

[31] M. Kontsevich, Enumeration of rational curves via torus actions, The Moduli Space of Curves (R. Dijkgraaf, C. Faber, and G. van der Geer, eds.), Progress in Math., vol. 129, Birkhäuser, 1995, pp. 335-368, hep-th/9405035.

[32] M. Kontsevich and Yu. Manin, Gromov-Witten classes, quantum cohomology, and enumerative geometry, Comm. Math. Phys. 164 (1994), 525-562, hep-th/9402147.

[33] W. Lerche, C. Vafa, and N. P. Warner, Chiral rings in $N=2$ superconformal theories, Nuclear Phys. B 324 (1989), 427-474.

[34] J. Li and G. Tian, Virtual moduli cycles and Gromov-Witten invariants of algebraic varieties, alg-geom/9602007.

[35] Yu. I. Manin, Generating functions in algebraic geometry and sums over trees, The Moduli Space of Curves (R. Dijkgraaf, C. Faber, and G. van der Geer, eds.), Progress in Math., vol. 129, Birkhäuser, 1995, pp. 401-417, alg-geom/9407005.

[36] D. McDuff and D. Salamon, J-holomorphic curves and quantum cohomology, University Lecture Series, vol. 6, American Mathematical Society, 1994.

[37] R. C. McLean, Deformations of calibrated submanifolds, (based on a 1991 Duke University Ph.D. thesis), Comm. Anal. Geom., to appear, preprint available at http://www.math.duke.edu/preprints/1996.html.

[38] D. R. Morrison, Compactifications of moduli spaces inspired by mirror symmetry, Journées de Géométrie Algébrique d'Orsay (Juillet 1992), Astérisque, vol. 218, Société Mathématique de France, 1993, pp. 243-271, alg-geom/9304007.

[39] _ Beyond the Kähler cone, Proc. of the Hirzebruch 65 Conference on Algebraic Geometry (M. Teicher, ed.), Israel Math. Conf. Proc., vol. 9, Bar-Ilan University, 1996, pp. 361-376, alg-geom/9407007.

[40] _ Mirror symmetry and moduli spaces of superconformal field theories, Proc. Internat. Congr. Math. Zürich 1994 (S. D. Chatterji, ed.), vol. 2, Birkhäuser Verlag, 1995, pp. 1304-1314, alg-geom/9411019.

[41] _ Making enumerative predictions by means of mirror symmetry, Mirror Symmetry II (B. Greene and S.-T. Yau, eds.), International Press, Cambridge, 1997, pp. 457-482, alg-geom/9504013.

[42] __ Mirror symmetry and the type II string, Trieste Conference on S-Duality and Mirror Symmetry, Nuclear Phys. B Proc. Suppl., vol. 46, 1996, pp. 146-155, hep-th/9512016. 
[43] _ Mathematical aspects of mirror symmetry, Complex Algebraic Geometry (J. Kollár, ed.), IAS/Park City Mathematics Series, vol. 3, 1997, pp. 265-340, alg-geom/9609021.

[44] D. R. Morrison and M. R. Plesser, Summing the instantons: Quantum cohomology and mirror symmetry in toric varieties, Nuclear Phys. B 440 (1995), 279-354, hep-th/9412236.

[45] - Towards mirror symmetry as duality for two-dimensional abelian gauge theories, Trieste Conference on S-Duality and Mirror Symmetry, Nuclear Phys. B Proc. Suppl., vol. 46, 1996, pp. 177-186, hep-th/9508107.

[46] S. Mukai, Symplectic structure of the moduli space of sheaves on an abelian or K3 surface, Invent. Math. 77 (1984), 101-116.

[47] _ On the moduli space of bundles on K3 surfaces, I, Vector Bundles on Algebraic Varieties, Bombay 1984, Oxford University Press, 1987, pp. 341-413.

[48] H. Ooguri, Y. Oz, and Z. Yin, D-branes on Calabi-Yau spaces and their mirrors, Nuclear Phys. B, 477 (1996), 407-430, hep-th/9606112.

[49] Y. Ruan, Topological sigma model and Donaldson-type invariants in Gromov theory, Duke Math. J. 83 (1996), 461-500.

[50] Y. Ruan and G. Tian, A mathematical theory of quantum cohomology, J. Differential Geom. 42 (1995), 259-367.

[51] C. T. Simpson, Moduli of representations of the fundamental group of a smooth projective variety, I, Inst. Hautes Études Sci. Publ. Math. 79 (1994), 47-129.

[52] A. Strominger, Massless black holes and conifolds in string theory, Nuclear Phys. B 451 (1995), 96-108, hep-th/9504090.

[53] A. Strominger, S.-T. Yau, and E. Zaslow, Mirror symmetry is T-duality, Nuclear Phys. B. 479 (1996), 243-259, hep-th/9606040.

[54] C. Voisin, A mathematical proof of a formula of Aspinwall and Morrison, Compositio Math. 104 (1996), 135-151.

Department of Mathematics, Box 90320, Duke University, Durham, NC 27708-0320, USA

E-mail address: drm@math.duke.edu 